\title{
BMJ Open COVID-19 emergency response assessment study: a prospective longitudinal survey of frontline doctors in the UK and Ireland: study protocol
}

\author{
Tom Roberts (D) , ${ }^{1,2}$ Jo Daniels, ${ }^{3}$ William Hulme, ${ }^{4}$ Daniel Horner, ${ }^{1,5}$ \\ Mark David Lyttle (1) , ,6 Katie Samuel, ${ }^{7}$ Blair Graham, ${ }^{8,9}$ Robert Hirst, ${ }^{7}$ \\ Charles Reynard, ${ }^{10}$ Michael Barrett (i) ${ }^{11}$ Edward Carlton ${ }^{1,12}$
}

To cite: Roberts T, Daniels J, Hulme W, et al. COVID-19 emergency response assessment study: a prospective longitudinal survey of frontline doctors in the UK and Ireland: study protocol. BMJ Open 2020;10:e039851. doi:10.1136/ bmjopen-2020-039851

- Prepublication history for this paper is available online. To view these files, please visit the journal online (http://dx.doi org/10.1136/bmjopen-2020039851).

Received 28 April 2020 Revised 22 July 2020 Accepted 23 July 2020
Check for updates

(C) Author(s) (or their employer(s)) 2020. Re-use permitted under CC BY-NC. No commercial re-use. See rights and permissions. Published by BMJ.

For numbered affiliations see end of article.

Correspondence to

Dr Tom Roberts;

tomkieranroberts@gmail.com

\section{ABSTRACT}

Introduction The COVID-19 pandemic is putting an unprecedented strain on healthcare systems globally. The psychological impact on frontline doctors of dealing with the COVID-19 pandemic is currently unknown. This Iongitudinal professional survey aims to understand the evolving and cumulative effects of working during the COVID-19 outbreak on the psychological well-being of doctors working in emergency departments (ED), intensive care units (ICU) and anaesthetics during the pandemic.

Methods and analysis This study is a longitudinal questionnaire-based study with three predefined time points spanning the acceleration, peak and deceleration phases of the COVID-19 pandemic.

The primary outcomes are psychological distress and post-trauma stress as measured by the General Health Questionnaire-12 (GHQ-12) and Impact of Events ScaleRevised (IES-R). Data related to personal and professional characteristics will also be collected. Questionnaires will be administered prospectively to all doctors working in ED, ICU and anaesthetics in the UK and Ireland via existing research networks during the sampling period. Data from the questionnaires will be analysed to assess the prevalence and degree of psychological distress and trauma, and the nature of the relationship between personal and professional characteristics and the primary outcomes. Data will be described, analysed and disseminated at each time point; however, the primary endpoint will be psychological distress and trauma at the final time point.

Ethics and dissemination Ethical approval was obtained from the University of Bath, UK (ref: 4421), and Children's Health Ireland at Crumlin, Ethics Committee. Regulatory approval from the Health Regulation Authority (UK), Health and Care Research Wales (IRAS: 281944).

This study is limited by the fact that it focuses on doctors only and is survey based without further qualitative interviews of participants. It is expected this study will provide clear evidence of the psychological impact of COVID-19 on doctors and will allow present and future planning to mitigate against any psychological impact.

Trial registration number ISRCTN10666798.

\section{Strengths and limitations of this study}

- This longitudinal study will assess psychological well-being in frontline doctors, at three time points across the pandemic wave, providing novel data in this potentially at-risk group.

- Both the General Health Questionnaire-12 and the Impact of Events Scale-Revised have been previously used in infectious disease outbreaks to measure psychological distress and trauma response.

- Collection of data at the 'peak' phase, capturing the degree of distress and personal and professional factors associated with distress at a prime time point of maximal stress on frontline doctors.

- Predetermined data collection points are reliant on national reporting and may not accurately reflect local or regional variations in systems pressure.

\section{INTRODUCTION}

Severe acute respiratory syndrome virus covariant 2 (SARS-CoV-2) is a presumed zoonotic novel coronavirus that first emerged in the province of Hubei, China, during late 2019. ${ }^{1}$ Viral transmission is presumed to be via droplet spread and it multiplies in respiratory epithelium. Clinical manifestations of the resulting COVID-19 disease include bilateral interstitial pneumonia, acute respiratory distress syndrome and multiorgan dysfunction syndrome. ${ }^{2}$ Due to high transmissibility, hospitalisation rates, critical care requirements and mortality rate in elderly and vulnerable populations, COVID-19 has created a public health emergency ${ }^{3}$ and was declared a pandemic by the WHO on 11 March 2020. ${ }^{4}$

Clinicians in acute and critical healthcare services provide medical care at the point of highest risk of disease transmission, and frequently undertake aerosol-generating procedures which increase their exposure 
to SARS-CoV-2. During comparable infectious disease outbreaks such as SARS-CoV and Ebola, healthcare workers were over-represented in disease incidence and poor clinical outcomes. Such concerns relating to COVID-19 are reflected in experiences anecdotally reported from the international healthcare community. ${ }^{5}$

This study will focus on doctors and not the wider healthcare workforce. It is well documented that other professions are potentially impacted more by infectious disease outbreaks and by COVID-19. ${ }^{6}$ Discussions were held between the study team and representatives from the Royal College of Nursing UK and College of Paramedics UK about a combined study. It was agreed that due to the limited timescale to collect data during the acceleration phase and complexities around different working practices that delaying data collection to involve a wider cohort would threaten the viability of the study. This protocol was shared with the colleges to support their independent studies, as well as ongoing information sharing to support study implementation.

In the UK and Ireland, doctors working in emergency departments (ED), intensive care units (ICU) and anaesthetics will be responsible for the initial identification, management and ongoing treatment of patients presenting with COVID-19. In addition, many difficult decisions relating to treatment escalation and resource allocation for individual patients will be made by clinicians working in these key areas. Many doctors are likely to be redeployed to these clinical areas or asked to work beyond their level of seniority. In addition, these doctors are likely to be directly responsible for the care of colleagues and staff members with the infection.

Resources in these clinical areas are already stretched at baseline. Operational pressures within EDs, critical care settings and emergency anaesthetic provision have been severe and escalating over a period of many years. This is reflected in the time to complete care episodes and health outcomes, ${ }^{7}$ the impact of fatigue and burnout within anaesthesia and ICU training, ${ }^{8}$ and the UK and Ireland having some of the lowest numbers of critical care beds per 100000 of population in Europe. ${ }^{9}$ This has resulted in concerns regarding surge capacity of facilities to cope with a pandemic illness. ${ }^{10}$ The psychological, emotional and physical demands placed on an already overstretched workforce may therefore be substantial.

It is evident from a substantial body of research across disaster settings that there is often a significant and longlasting negative impact on the psychological well-being of clinicians involved. ${ }^{11}{ }^{12}$ Similar themes are also emerging from the COVID-19 pandemic in a cross-sectional survey undertaken in selected healthcare workers in China. ${ }^{6}$

Key factors in predicting psychological distress posttrauma span a range of domains and include preparedness and training, ${ }^{13-15}$ social and occupational support, ${ }^{13-16}$ risk exposure and threat to life, ${ }^{141617}$ self-isolation, ${ }^{141618}$ media use, ${ }^{19} 20$ negative affect following exposure, ${ }^{14} 16-18$ history of mental health problems and previous trauma. ${ }^{15} 1718$ Yet, these have largely been identified post hoc, in the aftermath of events and without prospective data collection or a comprehensive understanding of the relative impact of these factors as an event unfolds.

To date, no large-scale longitudinal studies have proposed to prospectively examine the psychological distress and trauma response in clinicians during the acceleration, peak and deceleration phase of the pandemic wave of COVID-19. This study aims to understand the evolving and cumulative effects of working in EDs, ICUs and anaesthesia during the COVID-19 outbreak, specifically seeking to understand key personal and professional factors which predict psychological distress in this cohort of frontline doctors.

\section{METHODS AND ANALYSIS}

The primary aim of this study is to assess the prevalence and degree of psychological distress and trauma in doctors providing frontline care during the acceleration, peak and deceleration phases of the COVID-19 pandemic, and furthermore establish which personal and professional factors are associated with psychological distress at these time points.

More specifically, the objectives are to:

1. Evaluate personal and professional factors contributing to psychological well-being at the acceleration, peak and deceleration phase of the pandemic.

2. Establish the incidence of self-reported COVID-19 infection and self-isolation among frontline doctors, and to evaluate any association with psychological wellbeing.

3. Assess regional and national variation of psychological distress and trauma in doctors within the UK and Ireland.

\section{Study design and conduct}

This prospective online longitudinal survey consists of three phases commensurate with the fluctuation of an initial pandemic wave of COVID-19 in the UK and Ireland. More specifically,

- Phase 1: Acceleration survey; administered at 0 month (March 2020).

- Phase 2: Peak survey; administered on day 7 following the pandemic peak, as defined by COVID-19-related hospital deaths, in the UK and Ireland.

- Phase 3: Deceleration survey; administered 30 days following the peak survey.

These three phases have been adapted from the Centre for Disease Control (CDC) 'Preparedness and Response Framework for Influenzae Pandemics' (figure 1). ${ }^{21}$

\section{Outcome measures}

The coprimary outcome measures will be GHQ-12 scores from Phase 1, 2 and 3 surveys, and the IES-R score in Phase 2 and 3 surveys.

The General Health Questionnaire-12 (GHQ-12) ${ }^{22}$ is a brief, validated, 12-item self-report measure devised to screen for psychological distress in the general 


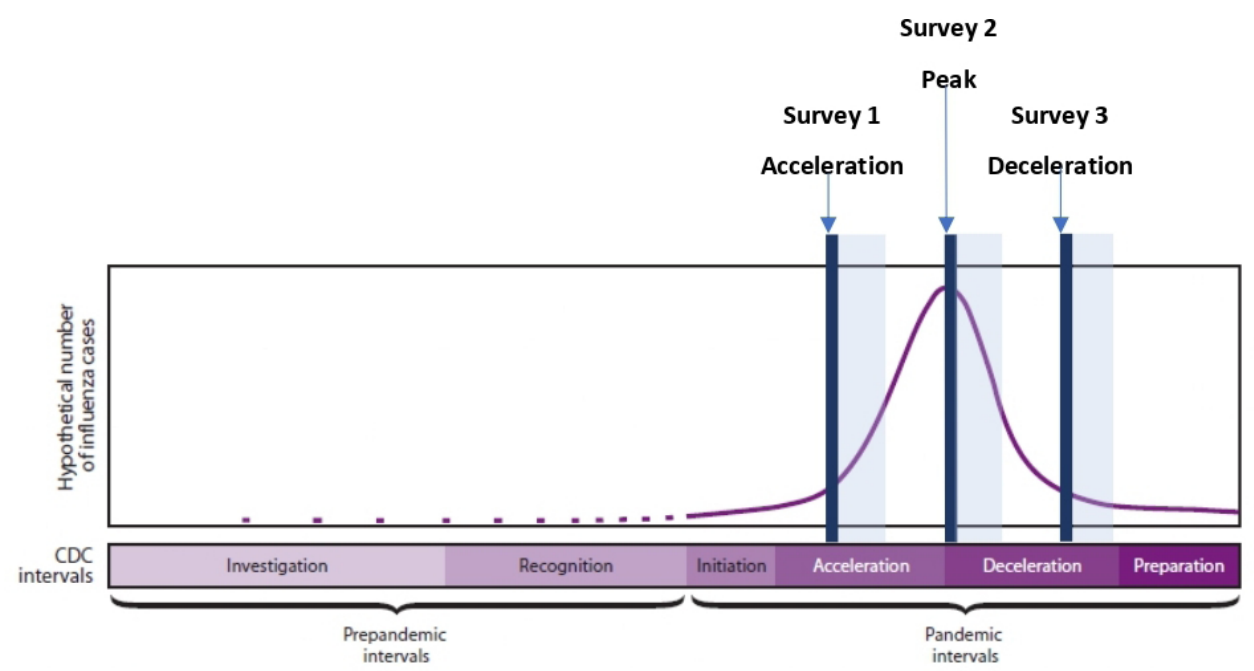

Figure 1 Timing of surveys in accordance with pandemic preparedness model. Solid blue line represents date of survey issue, transparent blue area represents data collection period (as adapted from the Centre for Disease Control (CDC) $)^{21}$.

population. It assesses current state (rather than longstanding attributes) and asks the participants to compare with the usual state. The measure has high specificity and sensitivity, with reliability demonstrated across a range of cultures and populations. ${ }^{23}$ The GHQ-12 has been used in similar clinician-based studies measuring the psychological impact of infectious outbreaks ${ }^{14}$ and was chosen due to the brevity of the measure and its suitability for time pressured medical staff. The GHQ-12 can be scored using several methods and we will report two of these in our results. The first, the 0-0-1-1 scoring method, is the most commonly used and has the highest sensitivity and specificity overall. ${ }^{23}$ This method has an established clinical cut-off of $>3$ which we will use to calculate prevalence of case level psychological distress in our study sample. ${ }^{23-25}$ The second uses a 0-1-2-3 scoring method which is sensitive to changes across time points; however, unlike the first method, there is no established cut-off and this technique reflects degree of distress rather than threshold caseness. We will use this method to detect within-person changes within our sample. By presenting the two different scoring methods, we can both report the prevalence of case level distress across the sample (0-0-1-1 scoring method) and detect changes within the sample over the three phases of the pandemic (0-1-2-3 scoring method).

The Impact of Events Scale-Revised (IES-R ${ }^{26}$ is a 22 -item measure commonly used to measure post-traumatic stress following a prespecified traumatic incident. Items are scored on a Likert scale, ranging from 0 representing 'not at all' to 4 representing 'extremely'. The IES-R has been commonly used in infectious disease outbreaks to assess post-traumatic stress in hospital staff. ${ }^{14}$ The IES-R has three subscales, relating to intrusion, avoidance and hyperarousal. Responses will be analysed similar to the GHQ-12, assigning the responses as $0,1,2,3,4$ (positive to negative) producing a score in the range $0-88$. A score of 24 or above will indicate a clinically significant stress response.

Secondary outcome measures will be predefined personal and professional characteristics (table 1) and their association with psychological distress as defined by GHQ-12 and IES-R.

\section{Participants}

Frontline medical staff employed in their main role as a doctor in the ED, ICU or anaesthetics in the UK and Ireland at the point of study commencement will be invited to participate. All grades of medical staff will be eligible to participate.

Doctors who move clinical setting between surveys will not be excluded, provided they remain within an acute trust setting. Doctors whose main place of employment at the point of study commencement is not the ED, ICU or anaesthetics and non-doctors working in ED, ICU or anaesthetics will be excluded. Participants will be asked to declare the hospital they work in. Hospitals will be grouped into regions as defined by UK Government coronavirus death reporting. ${ }^{27}$

\section{Survey distribution}

All potential participants will be invited to participate in the Phase 1 survey through established acute care research networks: in emergency medicine, members of the Trainee Emergency Research Network (TERN), Irish Trainee Emergency Research Network (I-TERN), Irish Association of Emergency Medicine and Paediatric Emergency Research in the UK and Ireland (PERUKI) will be invited to register as participating sites via institutional email and instant messaging groups. A site lead will be identified in each centre who will be responsible for distributing the participation link for the Phase 1 survey and encouraging participation through the display of relevant materials. In order to mitigate against non-UK or Ireland doctors and other healthcare groups completing 
Table 1 Personal and professional questions

\section{Demographic data}

Age

Gender

Ethnicity

\section{Employment-related factors}

Survey 1
$\checkmark$
$\checkmark$

Name of hospital

Parent specialty

Type of department

Redeployed to another clinical area

Where have you been redeployed to

How satisfied are you with this redeployment

Deployment back to original place of work

Local availability of psychological support

\section{Training and experience}

$\begin{array}{ll}\text { Survey } 1 & \checkmark \\ \checkmark & \text { Sur } \\ \checkmark & \\ \checkmark & \\ \checkmark & \checkmark \\ \checkmark & \checkmark \\ \checkmark & \checkmark\end{array}$

Previous infectious disease experience

Exposure to suspected/confirmed cases of COVID-19

Exposure to patients who have died due to suspected or confirmed COVID-19

Personal protective equipment training

Confidence in personal protective equipment training

COVID-19 practical clinical care training and confidence

Frequency of access and sources of clinical information

Perception of preparedness

Personal factors

Concern regarding worsening of mental health condition

Concern regarding worsening of physical health condition

Concerns about risk to personal health

Concerns about risk to family or loved ones

Experience of previous significant trauma (prior to COVID-19 pandemic)

Concern about risk of death to self

Perception of support from friends and family

Perception of support from senior leadership team

Perception of impact on other patient groups (not COVID-19)

Positive factors related to involvement with coronavirus response

\section{Personal experience of COVID-19}

Have you had to self-isolate

Reason for self-isolation

Number of clinical shifts missed due to self-isolation

\begin{tabular}{|c|c|c|c|}
\hline & Survey 1 & Survey 2 & Survey 3 \\
\hline & $\checkmark$ & $\checkmark$ & $\checkmark$ \\
\hline & $\checkmark$ & $\checkmark$ & $\checkmark$ \\
\hline & $\checkmark$ & $\checkmark$ & $\checkmark$ \\
\hline & & $\checkmark$ & $\checkmark$ \\
\hline us & & $\checkmark$ & $\checkmark$ \\
\hline & & & $s$ \\
\hline & & & $\checkmark$ \\
\hline ends & & $\checkmark$ & $\checkmark$ \\
\hline & & $\checkmark$ & $\checkmark$ \\
\hline
\end{tabular}

Have you been admitted to hospital due to coronavirus

Have you received an antibody test

What was the result of the antibody test

Any COVID-19-related illness or death in family or friends

Any COVID-19-related illness or death in colleagues

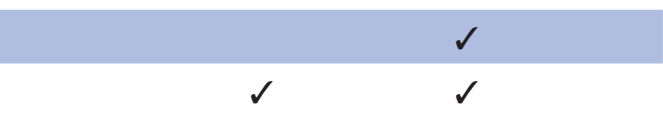

\section{Survey 1 Survey 2 Survey 3}

\section{$\checkmark$}

$\checkmark$

$\checkmark$

$\checkmark$

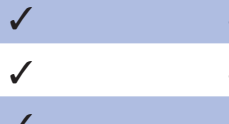

\section{Survey 1 Survey 2 Survey 3}

$\checkmark d$

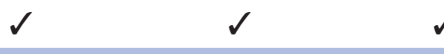

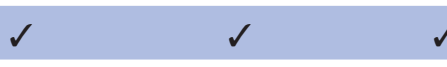

$\begin{array}{llll}2 & d & 2 & 2\end{array}$

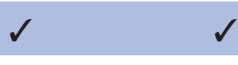

$\begin{array}{lll}2 & -1 \\ d & d\end{array}$

$\checkmark d$

$\begin{array}{lll}2 & 2 \\ d & d\end{array}$

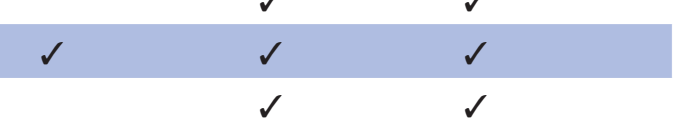

14 
the survey, the participation link will not be shared on wider social media platforms.

In the fields of intensive care and anaesthesia, participants will be invited to complete the Phase 1 Survey via the UK Research and Audit Federation of Trainees (RAFT) network membership groups and the Irish Specialist Anaesthesiology Trainee Audit \& Research Network (SATARN) via email and instant messaging. Additionally, participation invitations will be disseminated by the Royal College of Anaesthetists, College of Anaesthesiologists of Ireland and National Institute of Health Research (NIHR) Clinical Research Networks (including trauma and emergency care, critical care and anaesthesia and perioperative medicine) via email to regional leads, with additional invitations to all UK anaesthetists via the Lifelong Learning Platform. The Trainee Research in Intensive Care network will also distribute the survey link among their members and through the Faculty of Intensive Care Medicine.

\section{Survey design}

The survey has been designed and managed in line with the Checklist for Reporting Results of Internet E-surveys (CHERRIES) guidelines. ${ }^{28}$

A summary of survey construction is outlined in table 2. Each survey was developed iteratively by the study team and underpinned by evidence where available, or by consensus where necessary. Literature reviews were performed to identify factors with potential impact on psychological distress and trauma. Psychometric tools were selected by consensus of the study team, considering validity and utility of a range of measures, balanced against the feasibility of delivery and completion by individuals likely to be working at maximum capacity. Each survey will be piloted by members of the study team prior to full release.

\section{Phase 1: acceleration survey}

Phase 1 survey (online supplementary file 1) will gather consent and contact email address, selected personal and professional characteristics and responses to the GHQ-12 survey.

\section{Phase 2: peak survey}

All participants who completed the Phase 1 survey will be invited via the REDCap invite function to complete Phase 2 and 3 surveys. This uses a secure institutional email to deliver email invitations. The Phase 2 survey will gather consent and additional demographic, experiential or work-related data. No additional personal identifiable information will be taken. Participants will be requested to complete a serial evaluation of GHQ-12 and the IES-R; these are both valid and reliable short-form measures of their original counterparts and are used in order to limit participant fatigue.

Phase 3: deceleration survey

Phase 3 survey will gather consent and further data on personal and professional factors. No additional personal

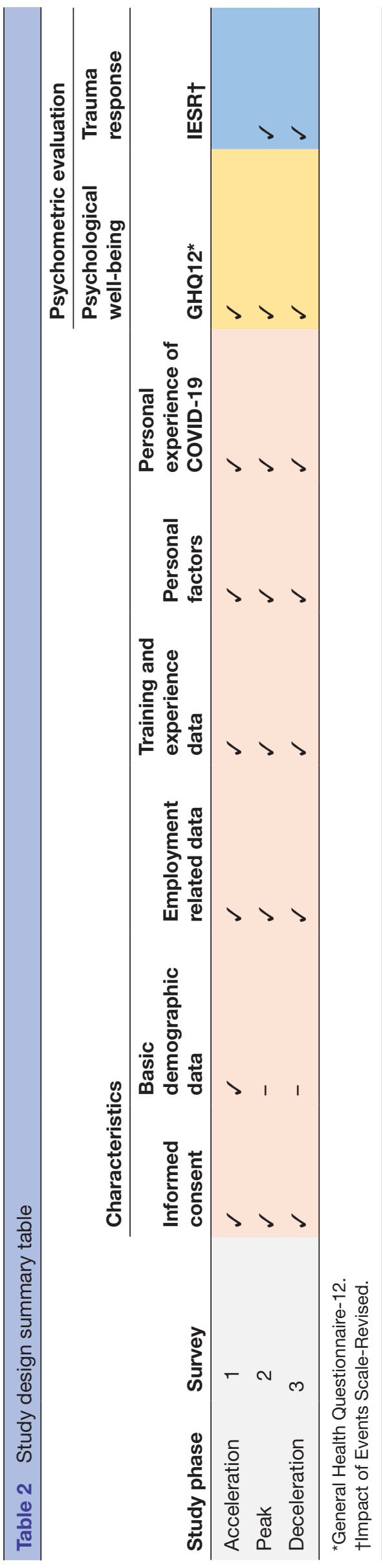


identifiable information will be taken, and it will be ensured that the survey does not exceed a reasonable length, to limit participant fatigue. Participants will be requested to complete a serial evaluation of GHQ-12 and IES-R.

\section{Survey timeline}

Identification of pandemic phases to guide survey release

The surveys will be released in keeping with the CDC pandemic framework outlined in figure 1. As the current outbreak is dynamic by its very nature, the exact timings of the peak and deceleration phases are uncertain but will be identified using the below criteria.

\section{Identification of acceleration phase}

The authors reached a consensus decision on 17 March 2020, based on best available evidence from Public Health England (PHE) that the UK was in the 'acceleration phase' of the current COVID-19 outbreak. Phase 1 survey was opened on 18 March 2020, for a period of 10 days.

\section{Identification of peak phase}

The authors will hold regular remote meetings to monitor the evolving COVID-19 outbreak. The 'Peak' survey will be released 7 days after the first $\mathrm{UK}$ and first Ireland national peaks of COVID-19-related deaths. The 7-day time delay is due to the requirement of the IES-R scale to reflect on feelings over the last 7 days, thus a delay will ensure that answers more accurately represent true outcomes from the pandemic peak. Nationally reported death rates have been chosen rather than confirmed cases due to a lack of consistency in screening and reporting of confirmed cases in the UK and Ireland. As UK national death rates are publicly available, in comparison to regional death rates, it is recognised that regional variation may occur.

The UK and Ireland national peaks will be decided by a consensus decision of the Study Management Group, which will be recorded and documented in the final study report. The consensus decision will be guided by:

- Publicly available COVID-19 daily death rates data from PHE (accessed via: https://coronavirus. data.gov.uk) and Ireland's Department of Health (accessed via: https://www.gov.ie/en/news/ 7e0924-latest-updates-on-covid-19-coronavirus/).

- Government daily briefings.

- Published modelling literature.

The survey will remain open for 14 days to ensure maximal response rates.

\section{Identification of deceleration phase}

The deceleration phase is defined by the CDC as 'consistently decreasing rate of cases'. ${ }^{21}$ To ensure the deceleration survey is released during this phase, it will be released 30 days after the administration of the 'Peak' survey. This is to ensure UK and Ireland cases are consistently decreasing and that there is no evidence of a second peak. The survey will remain open for 21 days.

\section{Informed consent}

Electronic informed consent will be obtained prior to completion of each round of the surveys.

\section{Withdrawal}

Participants can exit the survey online if they no longer wish to take part at any time. However, it will be clear in the introductory statement that data from questions already completed may be analysed.

\section{Administration}

The survey will be administered via the online platform REDCap. This electronic data capture platform is fully compliant with Good Clinical Practice, GDPR and 20 ISO 27001. It has stringent data security procedures and uses private servers. Data will be held securely on secure online server hosted by the University Hospitals Bristol and Weston NHS foundation Trust, UK.

\section{Patient and public involvement}

Staff well-being was rated the fourth highest priority of the James Lind Alliance Priority Setting Partnership, ${ }^{29}$ which involved extensive consultation with clinicians, patients, public and carers. This study does not directly involve patients; however, the potential impact that psychological trauma in doctors could have for patient care is concerning. Due to the urgency and unprecedented nature of the current situation, patient and public involvement directly related to this study has not been possible during the development of this protocol. It was felt inappropriate to seek stakeholder engagement from doctors over the short study development period as it could have detracted from pressing clinical demands.

\section{STATISTICAL ANALYSIS PLAN Response rate}

This will be presented using the CHERRIES checklist specifications. ${ }^{28}$ An overall response rate denominator will be reported using the data provided by the General Medical Council on doctors currently registered and working in ED, anaesthetics and ICU in the UK. Estimates on the denominator for participants from Ireland will be reported using data provided by individual hospital departments on doctors working in the ED, anaesthetics and ICU.

\section{Analysis cohort (inclusion/exclusion criteria)}

Non-consented, duplicate (by email address) and noncompletion of the minimum required dataset for analysis (completion of GHQ-12, grade and hospital) will be excluded. Duplicates are handled as follows: where two or more email addresses are present, the most complete survey will be taken. Note that a complete survey may include unanswered questions.

The primary analysis cohort will comprise participants who have completed the GHQ-12 in all three surveys and the IES-R in surveys 2 and 3. Subanalyses of completed 
Table 3 Threshold scores for the GHQ-12 and IES-R

Thresholds for clinical significance of each of the psychometric evaluations

$\begin{aligned} & \text { GHQ-12 } \\ & \text { General } \\ & \text { Function }\end{aligned}$
$\begin{aligned} & \text { Above } 3 \text { on the } 0-0-1-1 \text { scoring system } \\ & \text { represents case level psychological distress }\end{aligned}$
$\begin{aligned} & \text { IES-R } \\ & \text { Trauma }\end{aligned}$
$\begin{aligned} & 24 \text { or above on the } 0-1-2-3-4 \text { scoring } \\ & \text { system represents clinically significant stress } \\ & \text { response }\end{aligned}$

GHQ-12, General Health Questionnaire-12; IES-R, Impact of Events Scale-Revised.

surveys 1, 2 and 3, irrespective of completion of other survey, will also be reported.

Due to the difference in COVID-19-related policy between the Governments of the UK and Ireland, there may be a difference in timing of the pandemic wave. This could result in a significant difference of the study populations. Therefore, a study management group decision will be made, prior to final analysis, in regard to whether the difference of timing of the UK and Ireland's pandemic waves precludes joint analysis. Any decision will be documented in the final study report.

\section{Descriptive statistics}

Descriptive statistics relating to participants' personal and professional characteristics will be presented overall and by department/geographical region.

GHQ-12 items will be analysed both individually and aggregated into an overall score using the 0-1-2-3 method. This method assigns responses to $0,1,2,3$ (positive to negative sentiment) producing a score in the range 0 to 36 , with 0 representing the most healthy response and 36 the most unhealthy. Note that for case identification, the 0-0-1-1 method is used (see the Outcome measures section and table 3 ).

IES-R responses will be analysed similarly by assigning the responses to $0,1,2,3,4$ (positive to negative), producing a score in the range of $0-60$.

The distribution of GHQ-12 and IES-R scores will be presented graphically, with an appropriate measure of central tendency and variation provided. Comparisons between different personal and professional characteristics will also be made. Distributional (median, Q1, Q3) and mean differences will be reported. Proportions of respondents meeting thresholds of clinically significant impairment will be derived for each of the psychometric measures, as outlined in table 3.

These descriptive analyses will be performed for the primary analysis cohort and the survey-specific subcohorts. Participant dropout rates from survey 1 to surveys 2 and 3 will be reported.

\section{Intersurvey analysis}

The models outlined are descriptive, with model parameters intended to summarise observed statistical relationships rather than estimating underlying causal effects.
No formal null hypothesis significance testing will be performed to determine the presence or absence of statistically significant effect sizes, although $\mathrm{p}$ values for model estimates will be reported for reference.

\section{Change in the GHQ-12 score}

The change over time in the GHQ-12 score among participants who responded to all three surveys will be examined. Graphical relationships between the trend in the GHQ-12 score and variables collected at Phase 1 survey will be presented.

A repeated measures non-linear mixed effect model will be deployed. The dependent variable, GHQ score as measured on three consecutive occasions, is indexed either by survey response date (in continuous time) or by survey epidemic phase (before, during and after the epidemic peak). Models based on both indices will be investigated.

For the time-indexed model, a quadratic relationship between time and GHQ will be permitted (given the potential for a rise then fall in GHQ-12 over the course of the epidemic).

Region-level random effects on the intercept and time will be included in both time-indexed and phase-indexed models, enabling regional differences in the modelled effect of phase/time on GHQ and IES-R scores to be (partially) accounted for. Hospital-level random effects may also be investigated, depending on the number of responses per hospital. While hospital-level random effects would more appropriately account for betweenhospital heterogeneity than region-level random effects, it is anticipated that some hospitals will only be represented by only a very small number of participants, which may cause problems for model identification.

To identify potential modifiers of GHQ-12-score change, further models each with a single additional covariate will be built, with the likelihood ratio used to assess the degree of improvement in the model.

\section{Impact of Events Scale-Revised}

The IES-R score among participants who responded to all three surveys will be examined. Graphical relationships between the IES-R score and variables collected at survey 1 will be presented.

A linear model will be deployed seeking to account for the variation in the IES-R score with survey 1 variables.

To identify potential prepeak modifiers of IES-R-score (for instance, to identify characteristics that put clinicians at higher risk of trauma following an epidemic), further models each with a single additional covariate will be built, and a likelihood ratio test performed to assess the improvement in the model. For Phase 3 models, the IES-R score from Phase 2 will also be included as a covariate.

\section{Procedure for accounting for missing, unused and spurious data}

Information on completeness for each variable will be reported. For the primary models, missing values will be 
imputed using multilevel fully conditional specification multiple imputation with 100 imputed datasets to be created. ${ }^{30-32}$ For consistency, the same imputed datasets will be used across all models. Categorical variables will be imputed using multinomial logistic regression and ordinal variables using ordinal regression. The only continuous variables are GHQ-12 score and IES-R but these will be derived anew following imputation of the individual questions and will not be imputed directly. Imputation will not be necessary for region, grade and specialty as these are complete by design due to the exclusion criteria. An 'impute-then-delete' strategy will be employed for the dependent variable. Effect estimates across imputed datasets will be pooled using Rubin's rules. ${ }^{33}$

\section{Software}

All analyses and statistical outputs will be produced in the statistical programming language $\mathrm{R}$. The lme4 package will be used for the mixed-effects models.

\section{Procedures for reporting any deviation(s) from the original statistical plan}

Any requirement to deviate from the original statistical plan will be discussed with the Study Management Group and independently reviewed by an external statistician, where appropriate, and documented appropriately with a full explanation as to reasoning and requirement.

\section{Data storage}

Data will be stored electronically for 5 years by the University Hospital of Bristol and Weston NHS Foundation Trust.

\section{ETHICS AND DISSEMINATION \\ Ethical approval}

This project has ethical approval from the University of Bath, UK, and Children's Health Ireland at Crumlin, Ethics Committee (online supplementary 2). Regulatory approval was obtained from the Health Regulation Authority (UK), Health and Care Research Wales (online supplementary 3$)$.

\section{Risk to participants}

This survey collects potentially sensitive information, which will be handled in accordance with the General Data Protection Regulations. This includes details on participants' baseline health status and psychometric evaluations of anxiety, depression and post-traumatic stress. It will be emphasised in the participant information sheet that such measures are non-diagnostic and that the purpose of the study is to monitor psychological wellbeing on a population level. As scales are being used for non-diagnostic purposes, feedback will not be provided to participants regarding their scores. Participants will be given the option to not disclose existing physical or mental health complaints with these questions listed as 'optional'. It is possible that questions relating to personal health and well-being may trigger emotive responses in participants. Participants will be signposted to suggested local and national sources in the UK and Ireland where they may obtain support at the beginning and end of each survey.

\section{Risk to investigators}

There are no anticipated additional risks to investigators as part of this study. The study may generate media interest. All media releases will be conducted through the sponsor and/or publishing journals. Media interviews will be undertaken by a senior member of the study group with media training.

\section{Dissemination}

Interim study reports will be prepared for public dissemination. On study completion, a final manuscript will be submitted to a peer-reviewed scientific journal and shared with Medical Royal Colleges to inform stakeholders of the pandemic impact on this critical workforce. The results will be disseminated widely at scientific conferences.

\section{DISCUSSION}

This large-scale prospective longitudinal survey of frontline doctors builds on previous work regarding psychological well-being in acute care settings and looks to assess the psychological impact of the COVID-19 pandemic on frontline doctors, specifically seeking to understand key personal and professional factors which predict psychological distress in this cohort. Findings will be discussed in relation to the current context and in light of the reported impact of previous infectious disease outbreaks, aiming to contribute to novel data on frontline doctors' mental health in a rapidly emerging field.

Concerns have been raised regarding the potential and likely negative psychological impact of increasing workload in the already stretched ED clinical environment, with anticipation that this will be exacerbated by the specific and significant challenges of work during the COVID-19 pandemic. ${ }^{34}{ }^{35}$ In line with previous research, frontline healthcare workers are likely to be affected by fears of contamination, disruption of normal supportive structures and work stress. ${ }^{36}$ However, there is a paucity of data to quantify these effects. This collaborative research project, which harnesses the extensive reach of research networks, and supported by national professional bodies (such as the Medical Royal Colleges), seeks to address an important research question through rapid mobilisation of existing research infrastructures. The immediate outputs of this work will aim to inform the psychological response to this infection wave and future infection waves by robustly assessing the degree of psychological distress and trauma in the frontline workforce, furthermore gaining a greater understanding of the potentially modifiable personal and professional factors that predict distress. Establishing need is imperative given that trauma and psychological distress has been repeatedly demonstrated to have negative impact on occupational performance, job satisfaction, physical and psychological. ${ }^{37-39}$ 
By robustly identifying predictive factors associated with mental health outcomes in this population, targets for intervention will be provided; treatment for trauma and psychological distress is evidence-based, efficacious and widely available on the NHS. ${ }^{40}$ Recent advancements in psychological therapy provision have expanded adaptations for the frontline staff workforce ${ }^{41}$; however, there is currently a lack of knowledge concerning the precise prevalence and degree of distress and what characterises those who are most affected. This knowledge is essential to enable tailoring of support, treatment and pathways appropriate to the need. This research aims to address that gap and provide a foundation from which to shape service development in order to improve outcomes in this critical workforce.

The primary limitation to this work lies in estimating the peak phase and therefore the time point of maximal stress on frontline doctors. This is reliant on national reporting and may not reflect local or regional variations in systems pressure. However, given the high response rate and sample size in the acceleration phase survey, it is planned to mitigate regional effects through predefined subgroup analysis. Due to the rapidly developing nature of the pandemic, constraints have prevented the gathering of qualitative data as part of this study. Further research should explore the nature of distress in this population, drawing out themes that would enhance depth of knowledge in this area. There is a risk of selection bias through participant dropout from survey 1 to surveys 2 and 3. To mitigate against this, the GHQ-12 and IES-R results for those who drop out will be presented in the final analysis.

A further limitation to this work is the lack of baseline level of distress or trauma in this cohort prior to the COVID-19 pandemic. Work within the ED, ICU and anaesthetics is already known to be challenging and impact doctors' psychological health. ${ }^{842}{ }^{43}$ Results of this study will be presented in the context of the existing literature predating the COVID-19 pandemic.

In conclusion, this longitudinal professional survey aims to robustly assess the psychological impact of the COVID-19 pandemic on frontline doctors using sequential assessment to assess prevalence and degree of psychological distress across three key time points, defining the nature of the relationship between key personal and professional factors and primary outcomes of psychological distress and trauma response. This information will provide vital understanding of the impact of the COVID-19 pandemic on healthcare and well-being among clinical responders which will help tailor interventions and provide data for future planning of psychological support.

\section{Author affiliations}

${ }^{1}$ Trainee Emergency Research Network, The Royal College of Emergency Medicine, London, UK

${ }^{2}$ Emergency Department, Bristol Royal Hospital for Children, Bristol, UK

${ }^{3}$ Department of Psychology, University of Bath, Bath, UK

${ }^{4}$ Statistical Consultant, Oxford, UK

${ }^{5}$ Department of Intensive Care, Salford Royal Hospitals NHS Trust, Salford, UK
${ }^{6}$ Faculty of Health and Applied Science, University of the West of England, Bristol, UK ${ }^{7}$ Department of Anaesthesia, North Bristol NHS Trust, Westbury on Trym, UK ${ }^{8}$ Faculty of Health, University of Plymouth, Plymouth, UK

${ }^{9}$ Emergency Department, Plymouth Hospitals NHS Foundation Trust, Plymouth, UK

${ }^{10}$ Department of Cardviovascular Sciences, The University of Manchester,

Manchester, UK

${ }^{11}$ School of Medicine, Women's and Children's Health, University College Dublin, Dublin, Ireland

${ }^{12}$ Emergency Department, North Bristol NHS Trust, Westbury on Trym, UK

Twitter Mark David Lyttle @mdlyttle, Katie Samuel @katie_samuel_, Michael Barrett @PEMDublin and Edward Carlton @eddcarlton

Acknowledgements The views expressed are those of the authors and not necessarily those of the NHS, the NIHR, the Department of Health or the Royal Colleges involved in survey distribution. The authors would like to acknowledge Mai Baquedano, at the University of Bristol, for her support with REDCap. The authors would finally like to acknowledge GL Assessments for providing the licence for the GHQ-12 free of charge.

Contributors TR conceived the idea for the study. TR,EC, JD, MDL and BG were responsible for the initial study design, which was refined with the help of Katie Samuel KS, CR, RH, MB and WH. Expert advice on psychological assessment scores was provided by JD. WH provided the statistical plan. TR led the dissemination of the study in UK Adult Emergency Departments, MDL led the dissemination of the study in UK and Ireland Paediatric Emergency Departments, KS led the dissemination of the study in UK Anaesthetic and ICU Departments, MB led the dissemination of the study in Ireland Emergency Departments, ICUs and Anaesthetic Departments. TR coordinated study setup, finalisation of the study surveys and finalisations of study protocols. All authors contributed to the final study design and protocol development, critically revised successive drafts of the manuscript and approved the final version. The study management group is responsible for the conduct of the study.

Funding The study has been awarded a competitive grant by the Royal College of Emergency Medicine (G/2020/1). The survey platform is provided courtesy of the University of Bristol. The chief investigator is directly funded as a research fellow by the Royal College of Emergency Medicine. The GHQ-12 is being used under licence from GL assessments; the fee for use of this instrument within all three surveys has been waived. Dr Carlton is a National Institute for Health Research Advanced Fellow.

\section{Competing interests None declared.}

Patient and public involvement Patients and/or the public were not involved in the design, or conduct, or reporting or dissemination plans of this research.

Patient consent for publication Not required.

Provenance and peer review Not commissioned; externally peer reviewed.

Open access This is an open access article distributed in accordance with the Creative Commons Attribution Non Commercial (CC BY-NC 4.0) license, which permits others to distribute, remix, adapt, build upon this work non-commercially, and license their derivative works on different terms, provided the original work is properly cited, appropriate credit is given, any changes made indicated, and the use is non-commercial. See: http://creativecommons.org/licenses/by-nc/4.0/.

\section{ORCID iDs}

Tom Roberts http://orcid.org/0000-0003-4991-974X

Mark David Lyttle http://orcid.org/0000-0002-8634-7210

Michael Barrett http://orcid.org/0000-0003-1775-8347

\section{REFERENCES}

1 Chen N, Zhou M, Dong X, et al. Epidemiological and clinical characteristics of 99 cases of 2019 novel coronavirus pneumonia in Wuhan, China: a descriptive study. Lancet 2020;395:507-13.

2 Guan W, Ni Z, Hu Y, et al. Clinical characteristics of coronavirus disease 2019 in China. N Engl J Med 2020:NEJMoa2002032.

3 Mahase E. China coronavirus: who declares international emergency as death toll exceeds 200. BMJ 2020;368:m408.

4 WHO. Director-General's opening remarks at the media briefing on COVID-19 - 11 March, 2020. Available: https://www.who.int/ $\mathrm{dg} /$ speeches/detail/who-director-general-s-opening-remarks-atthe-media-briefing-on-covid-19-11-march-2020 [Accessed 31 Mar 2020]. 
5 Chang D, Xu H, Rebaza A, et al. Protecting health-care workers from subclinical coronavirus infection. Lancet Respir Med 2020;8:e13.

6 Lai J, Ma S, Wang Y, et al. Factors associated with mental health outcomes among health care workers exposed to coronavirus disease 2019. JAMA Netw Open 2020;3:e203976.

7 The Nuffield Trust. What's behind the A\&E "crisis"? Available: https:// www.nuffieldtrust.org.uk/resource/what-s-behind-the-a-e-crisis [Accessed 31 Mar 2020].

8 Looseley A, Wainwright E, Cook TM, et al. Stress, burnout, depression and work satisfaction among UK anaesthetic trainees; a quantitative analysis of the satisfaction and wellbeing in anaesthetic training study. Anaesthesia 2019;74:1231-9.

9 Bittner M-I, Donnelly M, van Zanten AR, et al. How is intensive care reimbursed? A review of eight European countries. Ann Intensive Care 2013;3:37.

10 Ferguson N, Laydon D, Nedjati- Gilani G, et al. Impact of nonpharmaceutical interventions (NPIs) to reduce COVID19 mortality and healthcare demande., 2020. Available: https://www.imperial.ac.uk/ media/imperial-college/medicine/sph/ide/gida-fellowships/ImperialCollege-COVID19-NPI-modelling-16-03-2020.pdf

11 Naushad VA, Bierens JJ, Nishan KP, et al. A systematic review of the impact of disaster on the mental health of medical responders. Prehosp Disaster Med 2019;34:632-43.

12 Lin $\mathrm{C}-\mathrm{Y}$, Peng Y-C, Wu Y-H, et al. The psychological effect of severe acute respiratory syndrome on emergency department staff. Emerg Med J 2007;24:12-17.

13 Brooks SK, Dunn R, Sage CAM, et al. Risk and resilience factors affecting the psychological wellbeing of individuals deployed in humanitarian relief roles after a disaster. $J$ Ment Health 2015;24:385-413.

14 Brooks SK, Dunn R, Amlôt R, et al. A systematic, thematic review of social and occupational factors associated with psychological outcomes in healthcare employees during an infectious disease outbreak. J Occup Environ Med 2018;60:248-57.

15 Lancee WJ, Maunder RG, Goldbloom DS, et al. Prevalence of psychiatric disorders among Toronto Hospital workers one to two years after the SARS outbreak. Psychiatr Serv 2008;59:91-5.

16 Maunder RG, Lancee WJ, Rourke S, et al. Factors associated with the psychological impact of severe acute respiratory syndrome on nurses and other Hospital workers in Toronto. Psychosom Med 2004:66:938-42.

17 Ozer EJ, Best SR, Lipsey TL, et al. Predictors of posttraumatic stress disorder and symptoms in adults: a meta-analysis. Psychol Bull 2003;129:52-73.

18 Brooks SK, Webster RK, Smith LE, et al. The psychological impact of quarantine and how to reduce it: rapid review of the evidence. Lancet 2020.

19 Jones NM, Thompson RR, Dunkel Schetter C, et al. Distress and rumor exposure on social media during a campus lockdown. Proc Natl Acad Sci U S A 2017;114:11663-8.

20 Torales J, O'Higgins M, Castaldelli-Maia JM, et al. The outbreak of COVID-19 coronavirus and its impact on global mental health. Int $J$ Soc Psychiatry 2020;66:317-20.

21 Holloway R, Rasmussen SA, Zaza S, et al. Updated preparedness and response framework for influenza pandemics, 2014. Available: https://www.cdc.gov/mmwr/preview/mmwrhtml/rr6306a1.htm [Accessed 8 Apr 2020].

22 Goldberg D, Williams P. A user's guide to the General Health Questionnaire. London: GL Assessment, 1988.
23 Goldberg DP, Gater R, Sartorius N, et al. The validity of two versions of the GHQ in the who study of mental illness in general health care. Psychol Med 1997;27:191-7.

24 Goldberg DP, Oldehinkel T, Ormel J. Why GHQ threshold varies from one place to another. Psychol Med 1998.

25 Burbeck R, Coomber S, Robinson SM, et al. Occupational stress in consultants in accident and emergency medicine: a national survey of levels of stress at work. Emerg Med J 2002;19:234-8.

26 Horowitz M, Wilner N, Alvarez W. Impact of event scale: a measure of subjective stress. Psychosom Med 1979;41:209-18.

27 Coronavirus. (COVID-19) in the UK. Available: https://coronavirus. data.gov.uk/\#category=regions\&map=rate [Accessed 17 Jun 2020].

28 Eysenbach $\mathrm{G}$. Improving the quality of web surveys: the checklist for reporting results of Internet E-Surveys (cherries). J Med Internet Res 2004;6:e34.

29 Smith J, Keating L, Flowerdew L, et al. An emergency medicine research priority setting partnership to establish the top 10 research priorities in emergency medicine. Emerg Med J 2017;34:454-6.

30 Marshall A, Altman DG, Holder RL, et al. Combining estimates of interest in prognostic modelling studies after multiple imputation: current practice and guidelines. BMC Med Res Methodol 2009;9:57.

31 Huque MH, Carlin JB, Simpson JA, et al. A comparison of multiple imputation methods for missing data in longitudinal studies. BMC Med Res Methodol 2018;18:168.

32 Grund S, Lüdtke O, Robitzsch A. Multiple imputation of missing data for multilevel models. Organ Res Methods 2018;21:111-49.

33 von Hippel PT. 4. regression with missing Ys: an improved strategy for analyzing multiply Imputed data. Sociol Methodol 2007;37:83-117.

34 Godlee F. Covid-19: weathering the storm. BMJ 2020;368:m1199.

35 BMA. BMA survey finds doctors' lives still at risk despite PPE pledges. Available: https://www.bma.org.uk/news-and-opinion/bmasurvey-finds-doctors-lives-still-at-risk-despite-government-pledgeson-ppe [Accessed 23 Apr 2020].

36 Holmes EA, O'Connor RC, Perry VH, et al. Multidisciplinary research priorities for the COVID-19 pandemic: a call for action for mental health science. Lancet Psychiatry 2020;7:547-60.

37 Maunder RG, Lancee WJ, Balderson KE, et al. LongTerm psychological and occupational effects of providing Hospital healthcare during SARS outbreak. Emerg Infect Dis 2006;12:1924-32.

38 Arora M, Asha S, Chinnappa J, et al. Review article: burnout in emergency medicine physicians. Emerg Med Australas 2013;25:491-5.

39 Fiksenbaum L, Marjanovic Z, Greenglass ER, et al. Emotional exhaustion and state anger in nurses who worked during the SARS outbreak: the role of perceived threat and organizational support. Can J Community Ment Heal 2006.

40 Pilling S, Whittington C, Taylor C, et al. Identification and care pathways for common mental health disorders: summary of NICE guidance. BMJ 2011;342:d2868.

41 BPS. The psychological needs of healthcare staff as a result of the coronavirus pandemic. Available: https://www.bps.org.uk/newsand-policy/psychological-needs-healthcare-staff-result-coronaviruspandemic [Accessed 23 Apr 2020].

42 Schneider A, Weigl M. Associations between psychosocial work factors and provider mental well-being in emergency departments: a systematic review. PLoS One 2018;13:e0197375.

43 Rotenstein LS, Torre M, Ramos MA, et al. Prevalence of burnout among physicians: a systematic review. JAMA 2018;320:1131-50. 